\title{
Scaling properties of bicritical dynamics in unidirectionally coupled period-doubling systems in the presence of noise
}

\author{
Kapustina, J.V.; Kuznetsov, A.P.; Kuznetsov, S.P.; Mosekilde, Erik
}

Published in:

Physical Review E. Statistical, Nonlinear, and Soft Matter Physics

Link to article, DOI:

10.1103/PhysRevE.64.066207

Publication date:

2001

Document Version

Publisher's PDF, also known as Version of record

Link back to DTU Orbit

Citation (APA):

Kapustina, J. V., Kuznetsov, A. P., Kuznetsov, S. P., \& Mosekilde, E. (2001). Scaling properties of bicritical dynamics in unidirectionally coupled period-doubling systems in the presence of noise. Physical Review E. Statistical, Nonlinear, and Soft Matter Physics, 64(6), 066207. https://doi.org/10.1103/PhysRevE.64.066207

\section{General rights}

Copyright and moral rights for the publications made accessible in the public portal are retained by the authors and/or other copyright owners and it is a condition of accessing publications that users recognise and abide by the legal requirements associated with these rights.

- Users may download and print one copy of any publication from the public portal for the purpose of private study or research.

- You may not further distribute the material or use it for any profit-making activity or commercial gain

- You may freely distribute the URL identifying the publication in the public portal 


\title{
Scaling properties of bicritical dynamics in unidirectionally coupled period-doubling systems in the presence of noise
}

\author{
Julia V. Kapustina, ${ }^{1,2}$ Alexandr P. Kuznetsov, ${ }^{1,2}$ Sergey P. Kuznetsov, ${ }^{1,2}$ and Erik Mosekilde ${ }^{3}$ \\ ${ }^{1}$ Institute of Radio-Engineering and Electronics RAS, Saratov Division, Zelenaya 38, Saratov, 410019, Russian Federation \\ ${ }^{2}$ Department of Nonlinear Processes, Saratov State University, Astrakhanskaja 83, Saratov, 410026, Russian Federation \\ ${ }^{3}$ Department of Physics, The Technical University of Denmark, $2800 \mathrm{Kgs}$. Lyngby, Denmark
}

(Received 21 May 2001; published 27 November 2001)

\begin{abstract}
We study scaling regularities associated with the effects of additive noise on the bicritical behavior of a system of two unidirectionally coupled quadratic maps. A renormalization group analysis of the effects of noise is developed. We outline the qualitative and quantitative differences between the response of the system to random perturbations added to the master subsystem or the slave subsystem. The universal constants determining the rescaling rules for the intensity of the noise sources in the master and slave subsystems are found to be $\gamma=6.619036 \ldots$ and $\nu=2.713708 \ldots$, respectively. A number of computer graphical illustrations for the scaling regularities is presented. We discuss the smearing of the fine structure of the bicritical attractor and the Fourier spectra in the presence of noise, the self-similar structure of the Lyapunov charts on the parameter plane near the bicritical point, and the shift of the threshold of hyperchaos in dependence of the noise intensity.

DOI: 10.1103/PhysRevE.64.066207

PACS number(s): 05.45.-a, 05.45.Df, 05.10.Cc
\end{abstract}

\section{INTRODUCTION}

Starting with the works of Feigenbaum [1], it has become clear that a description of the transition to chaos is not of qualitative nature only, but has a quantitative aspect as well. The problem is not restricted to enumerating the possible bifurcation scenarios on the road from regular to chaotic dynamics; there exist classes of quantitative universality associated with the distinct scenarios (at least, with some of them), which have to be revealed and studied. Systems of different natures (iterative maps, differential equations, delay differential equations, etc.) relating to a certain universality class demonstrate the same scaling regularities near the onset of chaos, characterized by definite universal scaling constants. The theoretical instrument for the analysis of these regularities is the renormalization group (RG) approach. Suggested first in the context of the period-doubling transition to chaos by Feigenbaum, this approach was extended by many authors in applications to other universality classes $[2,3]$.

In the context of a multiparameter analysis of the onset of chaos, a criticality of definite type may take place at certain surfaces, curves, or points in parameter space. RG analysis is an invaluable tool for the search, study, and classification of the critical situations. Their codimension (the number of parameters that have to be adjusted to reach criticality) is determined by the number of relevant unstable eigenvectors of the linearized RG equation (see, e.g., Ref. [3]).

Wishing to apply the concepts of universality and scaling to realistic systems, or to observe scaling regularities in a physical experiment, we must account for the presence of noise. Hence a question arises concerning the effects of noise on the dynamics near the critical points associated with the various universality classes.

Again, the deep and revealing approach to this problem is based on an appropriately modified version of the RG analysis. For the Feigenbaum criticality such an approach was first developed in the works of Crutchfield et al. and Shraiman et al. [4]. As shown by these authors, to observe one more level of period doubling one has to decrease the intensity of noise by a factor $\gamma=6.61903 \ldots$-the universal constant responsible for the scaling regularities of the effect of noise in the Feigenbaum universality class. Analogous analyses were undertaken, and the respective scaling constants were estimated for intermittency [5] and quasiperiodicity [6] in dissipative systems, as well as for period doubling [7] and KAM-torus destruction [8] in Hamiltonian systems. Nevertheless, other known universality classes, in particular associated with period doubling in multiparameter systems (see Ref. [3] for a review), have not been investigated with respect to the effects of noise.

An interesting class of dynamical models with nontrivial dynamics is represented by coupled maps with unidirectional coupling. In particular, these models were suggested to analyze and explain important peculiarities of turbulence in open flow systems [9]. More recently they were discussed in the context of applications to secure communication on a basis of the phenomenon of chaos syncronization [10]. In this paper we turn to a study of the effects of noise on the so-called bicritical behavior in a model of two onedimensional quadratic maps with unidirectional coupling [11-17]. The bicritical point corresponds to a situation when both subsystems are brought to the border of chaos by tuning of two parameters controlling the period doubling in these two maps. This may be regarded as the point of onset of hyperchaos in the unidirectionally coupled maps. Empirical (numerical and experimental) indications of the existence of this type of criticality and of the associated scaling regularities were presented in an earlier work [11]. Subsequently, a detailed theoretical study of the bicritical behavior was undertaken, accurate numerical values of the scaling constants were found, and explanations of the scaling properties of the phase space and parameter space were given on the basis of the $R G$ analysis $[12,3,13]$.

As it follows from the RG and universality arguments, a 


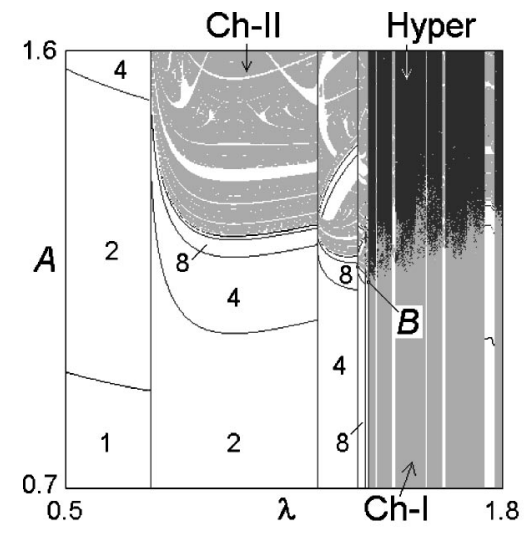

(a)

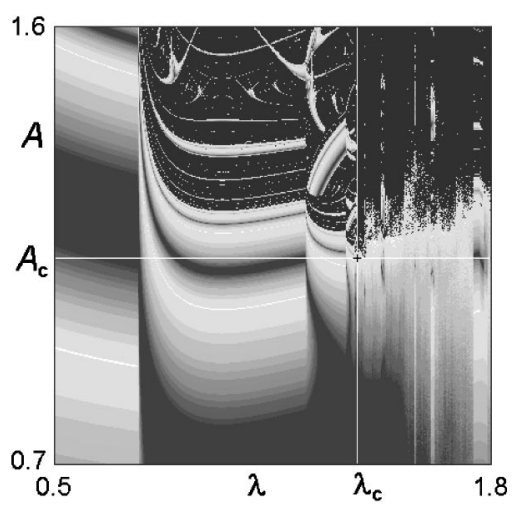

(b)

FIG. 1. Chart of the dynamical regimes or bifurcation diagram (a) and Lyapunov chart (b) for unidirectionally coupled maps [Eq. (1)] on the parameter plane $(\lambda, A)$ without noise; $B=0.375$. In panel (a) numbers indicate periods of regimes observed in the second subsystem. "Ch-I" is the regime of chaotic synchronization $\left(\Lambda_{1}>0, \Lambda_{2}<0\right.$ : chaos in the first subsystem induces the chaoslike motion of the second subsystem), "Ch-II" corresponds to chaotic motion only of the second subsystem $\left(\Lambda_{1}<0, \Lambda_{2}>0\right)$, and "Hyper" designates the regime of hyperchaos $\left(\Lambda_{1}>0, \Lambda_{2}>0\right)$. The bicritical point is marked by a letter $B$ on plot (a), and by a small cross in (b).

bicritical behavior may occur in systems of different nature, provided that they can be decomposed into two perioddoubling subsystems with unidirectional coupling. Besides the somewhat artificial model of coupled quadratic maps, the bicritical point was found numerically in more realistic models, such as two Chua circuits [14], driven nonlinear oscillators [15], and laser systems with unidirectional coupling [16]. Moreover, the bicritical behavior was observed in experiments with the unidirectionally coupled driven RL-diode circuits $[11,17]$. Obviously, a study of the effects of noise on the bicritical dynamics is of interest for the interpretation of these and other possible experiments. At the same time, it may be of importance for the application of the coupled map models, e.g., for a description of phenomena in open flows and for secure communication systems, where the effects of noise can be essential.

In Sec. II we introduce a model of two quadratic maps with unidirectional coupling, and recall a number of significant results known from previous studies. Then, in Sec. III, we add random perturbations to the model and present some numerical results to give a preliminary impression of the effects of noise. In particular, we obtain a rough estimate for a scaling constant associated with the noise. Section IV is devoted to an accurate RG analysis of the effects of noise on the bicritical behavior. We derive the RG equation, and present the results of a numerical solution including the improved high precision value for the universal constant. In Sec. V further conclusions following from the RG analysis are discussed, and computer illustrations for the scaling regularities are presented.

\section{A MODEL OF TWO QUADRATIC MAPS WITH UNIDIRECTIONAL COUPLING. THE BICRITICAL POINT}

Let us consider a system of two unidirectionally coupled quadratic maps $[12,3,13]$,

$$
x_{n+1}=1-\lambda x_{n}^{2}, \quad y_{n+1}=1-A y_{n}^{2}-B x_{n}^{2},
$$

where $x$ and $y$ are the dynamical variables for the first ("master") and second ("slave") subsystems, respectively; $\lambda$ and $A$ are parameters that control the period doubling in the subsystems, and $B$ is a coupling parameter. Figure 1(a) shows a chart of the dynamical regimes (a bifurcation diagram) of the model on the parameter plane $(\lambda, A)$ for $B=$ const. As the master system $x$ does not "feel" the dynamics of $y$, the bifurcations in that subsystem occur along vertical lines, corresponding to the bifurcation values of $\lambda$ for a single quadratic map. The period-doubling bifurcation lines converge, in accordance with the Feigenbaum law, to the critical line $\lambda=\lambda_{F}=1.401155 \ldots$ On the other hand, for any fixed $\lambda$ $<\lambda_{F}$ an increase of $A$ gives rise to a sequence of period doublings in the slave system. The cascade starts from a period corresponding to dynamics of the master system at the given $\lambda$, because this periodicity is induced in the second subsystem due to the coupling. The limit curve of the perioddoubling accumulation in the slave system $A=A_{F}(\lambda)$ meets the critical line of the master system at some point. This is what we call the bicritical point. In particular, for $B$ $=0.375$ it is located at

$$
\lambda_{c}=\lambda_{F}=1.401155189092 \ldots, \quad A_{c}=1.124981403 \ldots .
$$

The attractor at the bicritical point is a multifractal object embedded in two-dimensional phase space $(x, y)$. The selfsimilarity of the fractal structure near the origin is governed by the Feigenbaum constant $\alpha=-2.502907875 \ldots$ for $x$, and by a constant $\beta=-1.505318159 \ldots$ for $y$ [12]. The vicinity of the bicritical point is characterized by twodimensional scaling. One scaling factor coincides with that of Feigenbaum, $\delta_{1}=4.669201609 \ldots$ while the other is distinct, $\delta_{2}=2.39272443 \ldots$ [12]. Subsequent magnifications of the picture in a neighborhood of the bicritical point by 


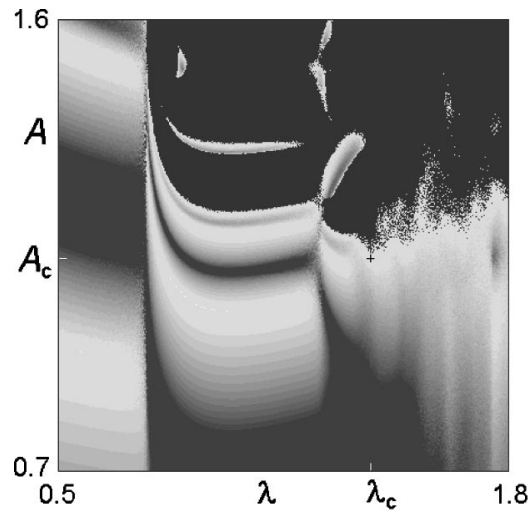

(a)

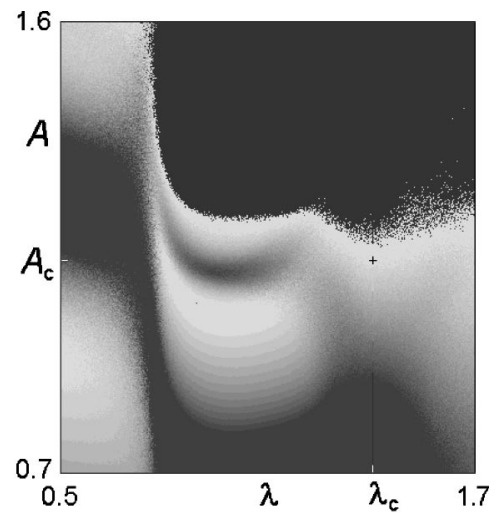

(b) factors $\delta_{1}$ and $\delta_{2}$ along the horizontal and vertical axes, respectively, allow the observation of a self-reproducing structure.

In fact, the bicritical situation takes place on a whole definite curve in the three-dimensional parameter space of model (1), so the same regularities can be observed for other positive values of the coupling parameter $B[13,18]$. For negative values of $B$ the bicritical behavior does not occur.

In Fig. 1(b) we depict the same area of parameter plane in the form of a Lyapunov chart (see Ref. [19] for previous applications of this method). For comparison, this is shown side by side with the chart of the dynamical regimes.

Our system has two Lyapunov exponents: $\Lambda_{1}=\Lambda_{1}(\lambda)$, which relates to the master system, and $\Lambda_{2}=\Lambda_{2}(\lambda, A, B)$, which relates to the slave system. This decomposition is possible, of course, due to the unidirectional nature of the coupling. At each pixel of the two-dimensional plot we estimate the Lyapunov exponent

$$
\Lambda_{2}=\lim _{N \rightarrow \infty}\left(\frac{1}{N} \sum_{n=1}^{N} \ln \left|2 A y_{n}\right|\right)
$$

from numerical computations, and mark the pixel by a gray tone. We code negative values of the Lyapunov exponent (from $-\infty$ to 0 ) by tones from dark to light gray. White represents zero values, and black denotes positive values of the Lyapunov exponent. This convention ensures a clear vision of the border between regular and chaotic dynamics. In contrast to the bifurcation diagram of Fig. 1(a), the Lyapunov chart retains a meaning in the presence of noise, so it will be useful in our further considerations.

It is worth noting that the bicritical behavior appears as well in a model with linear coupling [11]. Indeed, if the model map is chosen in the form

$$
x_{n+1}=1-\lambda x_{n}^{2}, \quad y_{n+1}=1-a y_{n}^{2}+\epsilon x_{n},
$$

then, substituting $X_{n}=x_{n-1}, Y_{n}=y_{n} /(1+\epsilon), A=a(1$ $+\epsilon), B=\epsilon \lambda /(1+\epsilon)$, we observe that $X_{n}, Y_{n}$ obey precisely to Eqs. (1). The bicritical behavior will take place at positive $\epsilon$.

\section{EFFECTS OF NOISE: EMPIRICAL RESULTS AND NUMERICAL ESTIMATE FOR THE NEW SCALING CONSTANT}

To account for noise in model (1) let us introduce two random sequences $\xi_{n}$ and $\eta_{n}$. We assume that $\xi_{n}$ and $\eta_{n}$ are statistically independent, and represent discrete-time white noise, i.e., the elements of each sequence at different steps of time are also independent. The maximal magnitudes of $\xi_{n}$ and $\eta_{n}$ are supposed to be bounded. The average for $\xi_{n}$ and $\eta_{n}$ is zero, $\left\langle\xi_{n}\right\rangle=\left\langle\eta_{n}\right\rangle=0$, and the standard deviation is some constant $\sigma,\left\langle\xi_{n} \xi_{n}\right\rangle=\left\langle\eta_{n} \eta_{n}\right\rangle=\sigma^{2}$, and crosscorrelations vanish, $\left\langle\xi_{n} \eta_{m}\right\rangle=0$ for any $n$ and $m$, and $\left\langle\xi_{n} \xi_{m}\right\rangle=\left\langle\eta_{n} \eta_{m}\right\rangle=0$ for $n \neq m$. Then we set

$$
\begin{gathered}
x_{n+1}=1-\lambda x_{n}^{2}+\kappa \xi_{n}, \\
y_{n+1}=1-A y_{n}^{2}-B x_{n}^{2}+\varepsilon \eta_{n},
\end{gathered}
$$

where $\kappa$ and $\varepsilon$ are parameters that characterize the intensity of the additive noise sources in the master and slave subsystems, respectively.

If the amplitude of noise is small, and the dynamics are considered on a large time scale, the concrete form of the probability distribution for $\xi_{n}$ and $\eta_{n}$ appears not to be essential, and the behavior of the noisy system will be of a universal nature (cf. Ref. [20]). In the present computations we define $\xi_{n}$ and $\eta_{n}$ as random values uniformly distributed over an interval $[-0.5,0.5]$. Hence, the standard deviation $\sigma=1 / \sqrt{12}$.

In Figs. 2 and 3 we present Lyapunov charts obtained in the presence of noise in only the first or in the second subsystem, respectively. As in Fig. 1(b), the gray tones code values of the Lyapunov exponent $\Lambda_{2}$ defined by formula (3). It is interesting to compare these diagrams with Fig. 1(b) to observe the increasing degree of smearing of the fine structures on the parameter plane as the noise intensity grows.

The clear difference between Figs. 2 and 3 indicates the essentially distinct character of the effects of noise on the master and slave subsystems. In the first case the smearing of the fine structure in the parameter plane looks more or less diffusivelike. In contrast, in the second case smearing takes places only along the vertical direction. Vertical borders, as- 


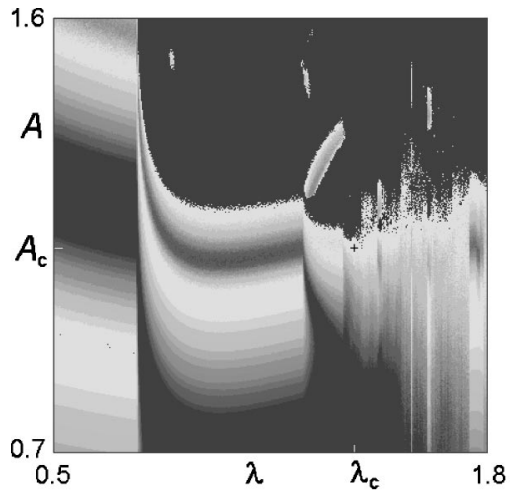

(a)

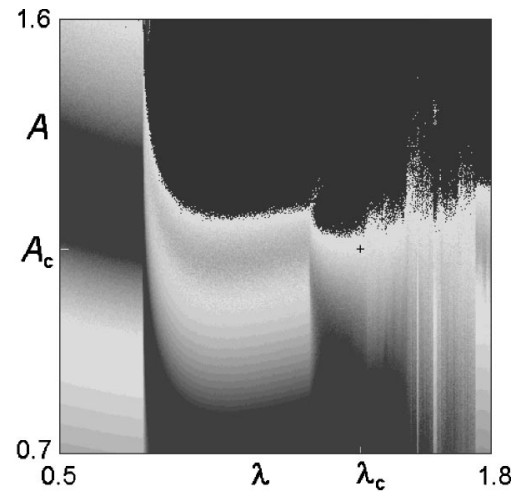

(b) sociated with the bifurcations in the master system, remain clearly visible.

In accordance with the results of Refs. [4], to observe each next level of period doubling in the master system we have to decrease the noise magnitude $\kappa$ by a factor $\gamma$ $=6.61903 \ldots$. It is natural to ask about an analogous rule for the case when the source of noise is placed in the slave system. To give a preliminary account and obtain a rough estimate of the associated scaling constant, in Fig. 4 we show the plots of the variable $y$ versus parameter $A$ in a vicinity of the bicritical point $\left[A_{\mathrm{c}}-\Delta A, A_{\mathrm{c}}+\Delta A\right]$. The other parameters are kept constant: $\lambda=\lambda_{c}=1.401155 \ldots$ and $B$ $=0.375$. These pictures are analogous to the commonly known Feigenbaum bifurcation diagrams (the bifurcation trees). However, here they are drawn for a slave map subjected to external driving (due to coupling with the master subsystem and due to the presence of a source of noise). The computations were performed with noise only in the slave subsystem, i.e., $\kappa=0$, while $\varepsilon$ is nonzero.

The top left panel of Fig. 4 corresponds to a definite arbitrarily chosen noise magnitude, $\varepsilon=\varepsilon_{0}=0.0005$. To make the structure distinguishable, we do not plot all values accepted by the variable $y$ in the process of dynamics, but only those values that occur with a step of $\Delta n=16$ iterations. The three other diagrams are obtained under rescaling through six period-doubling levels; that is, we increase the number of iterations and the step $\Delta n$ by a factor $2^{6}$, decrease the interval for $A$ by $\delta_{2}^{6}$, the interval for $y$ by $\beta^{6}$, and the magnitude of noise by some factor $\nu^{6}$. The constant $\nu$ should be selected to make the rescaled picture as similar as possible to the original. Setting $\nu=2.3,2.7$, and 3.1, we see that $\nu$ $=2.7$ seems to be an optimal value. For smaller $\nu$ the rescaled diagram looks more noisy, and for larger $\nu$ less noisy than the original.

A simple numerical procedure makes it possible to improve the estimate of the scaling constant. Let us turn to Eqs. (5), set $\kappa=0$, and assume $\varepsilon$ to be small. Then we can search for a solution in the form $y=Y+\varepsilon \tilde{y}$. Substituting this expression into Eqs. (5) and retaining terms up to the first order in $\varepsilon$, we have

$$
x_{n+1}=1-\lambda x_{n}^{2}, \quad Y_{n+1}=1-A Y_{n}^{2}-B x_{n}^{2}
$$

and

$$
\tilde{y}_{n+1}=-2 A Y_{n} \tilde{y}_{n}+\eta_{n} .
$$

Now we raise the last equation to the second power, and perform an averaging over the ensemble of samples for the random sequence $\eta_{n}$. This yields

$$
D_{n+1}=4 A^{2} Y_{n}^{2} D_{n}+1,
$$

where $D_{n}=\left\langle\tilde{y}_{n}^{2}\right\rangle / \sigma^{2}$. (Here we take into account that $\eta_{n}$ and $\tilde{y}_{n}$ are independent random variables by virtue of the whitenoise nature of $\eta_{n}$. Hence, the term $\left\langle 2 A Y_{n} \tilde{y}_{n} \eta_{n}\right\rangle$ disappears: $\left\langle 2 A Y_{n} \tilde{y}_{n} \eta_{n}\right\rangle=\left\langle 2 A Y_{n} \tilde{y}_{n}\right\rangle\left\langle\eta_{n}\right\rangle=0$.)

Now Eq. (8) together with Eq. (6) appear to be a closed set of equations that may be iterated numerically. We can take critical values for the parameters, $\lambda=\lambda_{c}$ and $A=A_{c}$, and perform $2^{k}$ iterations of Eqs. (6) and (8) starting from $x_{0}=0, Y_{0}=0$, and $D_{0}=0$. We may regard $\left|Y_{2^{k}}\right|$ as a characteristic scale for a displacement of $y$ of dynamical nature,

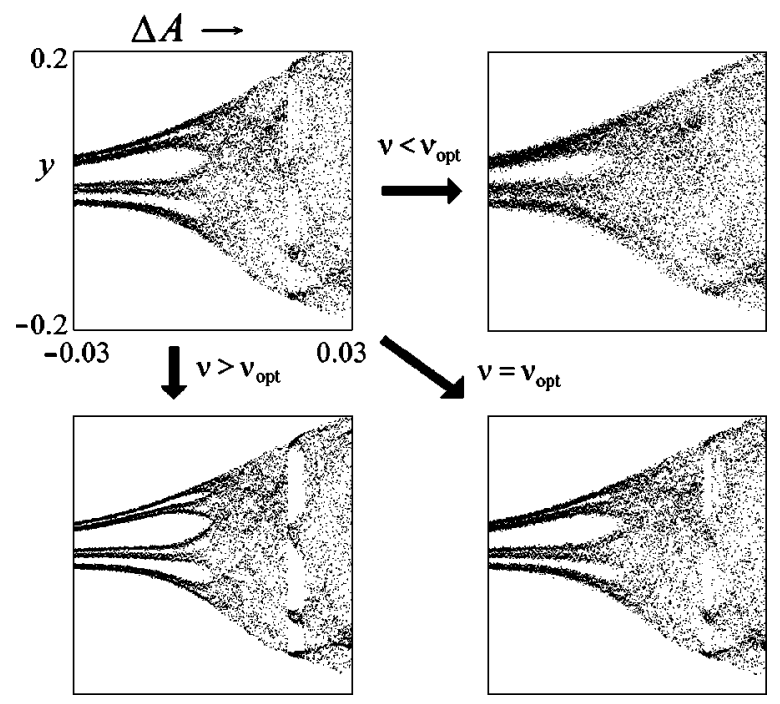

FIG. 4. Bifurcation tree in the presence of noise in the slave system: variable $y$ vs parameter $A$ at $\lambda=\lambda_{c}$. The first picture corresponds to a noise magnitude $\varepsilon=\varepsilon_{0}=0.0005$; the others are obtained after a rescaling through six levels, i.e., $\Delta A \rightarrow \Delta A / \delta_{2}^{6}, \Delta y$ $\rightarrow \Delta y / \beta^{6}$, and $\varepsilon \rightarrow \varepsilon / \nu^{6}$, where $\nu=2.3,2.7$, and 3.1, respectively. On the top left diagram each 16th iteration step for $y$ is plotted, on the other diagrams we plot each 1024th step. 
and $\sqrt{D_{2^{k}}}$ as a scale for a random displacement due to the noise. Their ratio $R_{k}=\sqrt{D_{2^{k}}}\left|Y_{2^{k}}\right|$ characterizes the relative effect of the noise on the dynamics. Comparing the values of $R_{k}$ at two subsequent levels we come to an estimate $\nu$ $\cong R_{k+1} / R_{k}$. Performing the computations for $k$ of order 10 13 , we find $\nu \cong 2.712 \pm 0.002$.

\section{RENORMALIZATION GROUP ANALYSIS OF BICRITICALITY IN THE PRESENCE OF NOISE}

Let us write out equations for two unidirectionally coupled noisy subsystems in following generalized forms

$$
\begin{gathered}
x_{n+1}=G\left(x_{n}\right)+\kappa U\left(x_{n}\right) \xi_{n}, \\
y_{n+1}=F\left(x_{n}, y_{n}\right)+\varepsilon V\left(x_{n}, y_{n}\right) \eta_{n}+\kappa W\left(x_{n}, y_{n}\right) \xi_{n} .
\end{gathered}
$$

Obviously, Eqs. (5) represent a particular version of this model with $G(x)=1-\lambda x^{2}, U(x)=1, F(x, y)=1-A y^{2}$ $-B x^{2}, V(x, y)=1$, and $W(x, y)=0$.

The stochastic map [Eqs. (9)] describes the evolution of the system over one step of discrete time. We can apply it twice to obtain the stochastic map for two steps of iteration. We suppose that the parameters of the noise magnitude are small $(\kappa, \varepsilon \ll 1)$ and neglect terms of second and higher orders. Then, using the rescaling $x \rightarrow x / \alpha, y \rightarrow y / \beta$, we have

$$
\begin{aligned}
x_{n+2}= & \alpha G\left(G\left(x_{n} / \alpha\right)\right)+\alpha \kappa\left[G^{\prime}\left(G\left(x_{n} / \alpha\right)\right) U\left(x_{n} / \alpha\right) \xi_{n}+U\left(G\left(x_{n} / \alpha\right)\right) \xi_{n+1}\right], \\
y_{n+2}= & \beta F\left(G\left(x_{n} / \alpha\right), F\left(x_{n} / \alpha, y_{n} / \beta\right)\right)+\beta \varepsilon\left[F_{y}^{\prime}\left(G\left(x_{n} / \alpha\right), F\left(x_{n} / \alpha, y_{n} / \beta\right)\right) V\left(x_{n} / \alpha, y_{n} / \beta\right) \eta_{n}\right. \\
& \left.+V\left(G\left(x_{n} / \alpha\right), F\left(x_{n} / \alpha, y_{n} / \beta\right)\right) \eta_{n+1}\right] \\
& +\beta \kappa\left[F_{x}^{\prime}\left(G\left(x_{n} / \alpha\right), F\left(x_{n} / \alpha, y_{n} / \beta\right)\right) U\left(x_{n} / \alpha\right) \xi_{n}+F_{y}^{\prime}\left(G\left(x_{n} / \alpha\right), F\left(x_{n} / \alpha, y_{n} / \beta\right)\right) W\left(x_{n} / \alpha, y_{n} / \beta\right) \xi_{n}\right. \\
& \left.+W\left(G\left(x_{n} / \alpha\right), F\left(x_{n} / \alpha, y_{n} / \beta\right)\right) \xi_{n+1}\right] .
\end{aligned}
$$

Accounting for the white-noise nature of the independent random variables $\xi$ and $\eta$, we may redefine the white-noise random processes to represent Eqs. (10) in forms analogous to Eqs. (9),

$$
\begin{gathered}
x_{n+2}=G_{1}\left(x_{n}\right)+\kappa U_{1}\left(x_{n}\right) \xi_{n}, \\
y_{n+2}=F_{1}\left(x_{n}, y_{n}\right)+\varepsilon V_{1}\left(x_{n}, y_{n}\right) \eta_{n}+\kappa W_{1}\left(x_{n}, y_{n}\right) \xi_{n},
\end{gathered}
$$

by an appropriate selection of the amplitude functions at the noise terms.

Next the same procedure may be applied to Eqs. (11) to obtain the stochastic map for four iterations, and so on. After $k$ steps we will have the maps for $2^{k}$ steps of discrete time,

$$
\begin{gathered}
x_{n+2^{k}}=G_{k}\left(x_{n}\right)+\kappa U_{k}\left(x_{n}\right) \xi_{n}, \\
y_{n+2^{k}}=F_{k}\left(x_{n}, y_{n}\right)+\varepsilon V_{k}\left(x_{n}, y_{n}\right) \eta_{n}+\kappa W_{k}\left(x_{n}, y_{n}\right) \xi_{n},
\end{gathered}
$$

where the functions obey the recurrent functional relations

$$
\begin{aligned}
G_{k+1}(x)= & \alpha G_{k}\left(G_{k}(x / \alpha)\right), \\
F_{k+1}(x, y)= & \beta F_{k}\left(G_{k}(x / \alpha), F_{k}(x / \alpha, y / \beta)\right), \\
U_{k+1}(x)=\mid & |\alpha|\left\{\left[G_{k}^{\prime}\left(G_{k}(x / \alpha)\right)\right]^{2}\left(U_{k}(x / \alpha)\right)^{2}\right. \\
& \left.+\left[U_{k}\left(G_{k}(x / \alpha)\right)\right]^{2}\right\}^{1 / 2},
\end{aligned}
$$

$$
\begin{aligned}
V_{k+1}(x, y)= & |\beta|\left\{\left[F_{k, y}^{\prime}\left(G_{k}(x / \alpha), F_{k}(x / \alpha, y / \beta)\right) V_{k}(x / \alpha, y / \beta)\right]^{2}+\left[V_{k}\left(G_{k}(x / \alpha), F_{k}(x / \alpha, y / \beta)\right)\right]^{2}\right\}^{1 / 2}, \\
W_{k+1}(x, y)= & |\beta|\left\{\left[F_{k, x}^{\prime}\left(G_{k}(x / \alpha), F_{k}(x / \alpha, y / \beta)\right) U_{k}(x / \alpha)\right.\right. \\
& \left.\left.+F_{k, y}^{\prime}\left(G_{k}(x / \alpha), F_{k}(x / \alpha, y / \beta)\right) W_{k}(x / \alpha, y / \beta)\right]^{2}+\left[W_{k}\left(G_{k}(x / \alpha), F_{k}(x / \alpha, y / \beta)\right)\right]^{2}\right\}^{1 / 2} .
\end{aligned}
$$

(Here the indices $x$ and $y$ denote the derivatives of $F$ with respect to the first or second argument of this function, respectively.)

As we take the original map at the bicritical point and set $\alpha=\alpha_{F}=-2.5029 \ldots$ and $\beta=-1.5053 \ldots$; then $G_{k}(x)$ and $F_{k}(x, y)$ converge to definite limit functions $g(x)$ and $f(x, y)$. These functions satisfy a set of functional equations

$$
\begin{gathered}
g(x)=\alpha g(g(x / \alpha)), \\
f(x, y)=\beta f(g(x / \alpha), f(x / \alpha, y / \beta)) .
\end{gathered}
$$

Equations (14) determine the fixed point of the RG transformation responsible for the universality class associated with the bicritical behavior [12,3]. The first line in Eqs. (14) 
obviously coincides with the well-known FeigenbaumCvitanović equation [1]. Here it is convenient to normalize $x$ and $y$ in such way that $g(0)=1$ and $f(0,0)=1$.

At large $k$ the functions $U_{k}(x)$ and $V_{k}(x, y)$ will behave asymptotically as

$$
U_{k}(x) \cong \sqrt{\Omega^{k} \Phi(x)}, \quad V_{k}(x, y) \cong \sqrt{\Theta^{k} \Psi(x, y)},
$$

where $\Omega=\gamma^{2}$ and $\Theta=\nu^{2}$ are the largest eigenvalues, and $\Phi$ and $\Psi$ the corresponding eigenfunctions for the linear functional equations:

$$
\Omega \Phi(x)=\alpha^{2}\left\{\left[g^{\prime}(g(x / \alpha))\right]^{2} \Phi(x / \alpha)+\Phi(g(x / \alpha))\right\},
$$

and

$$
\begin{aligned}
\Theta \Psi(x, y)= & \beta^{2}\left\{\left[f_{y}^{\prime}(g(x / \alpha), f(x / \alpha, y / \beta))\right]^{2} \Psi(x / \alpha, y / \beta)\right. \\
& +\Psi(g(x / \alpha), f(x / \alpha, y / \beta))\},
\end{aligned}
$$

respectively.

Equation (16) describes the effect of noise on the master system, and, of course, it coincides with the previously obtained expression for a single noisy period-doubling map [4]. As found numerically, the largest eigenvalue is

$$
\gamma=6.619036513 \ldots
$$

Figure 5(a) shows the eigenfunction $\Phi(x)$.

Equation (17) describes the effect of the noise source added to the slave system. It contains the universal functions $g(x)$ and $f(x, y)$ relating to the fixed point solution of the $\mathrm{RG}$ equation (14). These functions were computed in a form of polynomial expansions over even powers of the arguments [see Refs. [1,21,3] for the coefficients of expansion for $g(x)$, and Refs. $[12,3]$ for $f(x, y)]$. Using these data we have constructed the functional transformation of the right-hand side of Eq. (17) as a computer program. The unknown function $\Psi(x, y)$ is represented by a table of its values on a rectangular two-dimensional lattice in the square $-1 \leqslant x \leqslant 1,-1$ $\leqslant y \leqslant 1$ and by a fourth-order polynomial interpolation scheme between the lattice sites. Taking random initial conditions for $\Psi(x, y)$, we then perform a functional transformation and normalize the resulting function: $\Psi^{0}(x, y)$ $=\Psi(x, y) / \Psi(0,0)$. This operation is repeated many times, until the form of the function stabilizes, and the value of $\Psi(0,0)$ (before the normalization) becomes equal to $\Theta=\nu^{2}$. The result of these computations for the new scaling constant is

$$
\nu=2.713708 \ldots,
$$

which is in a reasonable agreement with the estimates of Sec. III. Figure 5(b) shows the three-dimensional plot for the eigenfunction $\Psi(x, y)$.

With respect to the last unknown function, $W_{k}(x, y)$, it follows from Eq. (13) that under subsequent RG transformation it will asymptotically behave in accordance with the equation

$$
\begin{aligned}
W_{k+1}(x, y)=\mid & \beta \mid\left\{\left[f_{x}^{\prime}(g(x / \alpha), f(x / \alpha, y / \beta)) U_{k}(x / \alpha)\right.\right. \\
& \left.+f_{y}^{\prime}(g(x / \alpha), f(x / \alpha, y / \beta)) W_{k}(x / \alpha, y / \beta)\right]^{2} \\
& \left.+\left[W_{k}(g(x / \alpha), f(x / \alpha, y / \beta))\right]^{2}\right\}^{1 / 2} .
\end{aligned}
$$

Without the term proportional to $U_{k}$, it takes the same form as the equation for the function $V_{k}$, so the asymptotic solution would behave as $\nu^{k}$. This component may be accounted for in the term $V_{k}$ in the stochastic map [Eq. (12)]. In presence of the term $U_{k}$ the solution is forced to behave as $\gamma^{k}$ (note that $\gamma>\nu$ ). So, the equation for $W$ does not provide new relevant scaling constants.

In a linear approximation with respect to the noise amplitude the stochastic map for the evolution over $2^{k}$ steps at the bicritical point is represented asymptotically as

$$
\begin{gathered}
x_{n+2^{k}}=g\left(x_{n}\right)+\kappa \gamma^{k} U\left(x_{n}\right) \xi_{n}, \\
y_{n+2^{k}}=f\left(x_{n}, y_{n}\right)+\varepsilon \nu^{k} V\left(x_{n}, y_{n}\right) \eta_{n}+\kappa \gamma^{k} W\left(x_{n}, y_{n}\right) \xi_{n} .
\end{gathered}
$$

If we consider a slight shift of parameters $\lambda$ and $A$ from the bicritical point then additional perturbation terms appear in the equation (see Ref. [12]):

$$
\begin{aligned}
x_{n+2^{k}}= & g\left(x_{n}\right)+C_{1} \delta_{1}^{k} h_{1}\left(x_{n}\right)+\kappa \gamma^{k} U\left(x_{n}\right) \xi_{n}, \\
y_{n+2^{k}}= & f\left(x_{n}, y_{n}\right)+C_{2} \delta_{2}^{k} h_{1}\left(x_{n}\right)+C_{1} \delta_{1}^{k} h_{12}\left(x_{n}\right) \\
& +\varepsilon \nu^{k} V\left(x_{n}, y_{n}\right) \eta_{n}+\kappa \gamma^{k} W\left(x_{n}, y_{n}\right) \xi_{n} .
\end{aligned}
$$

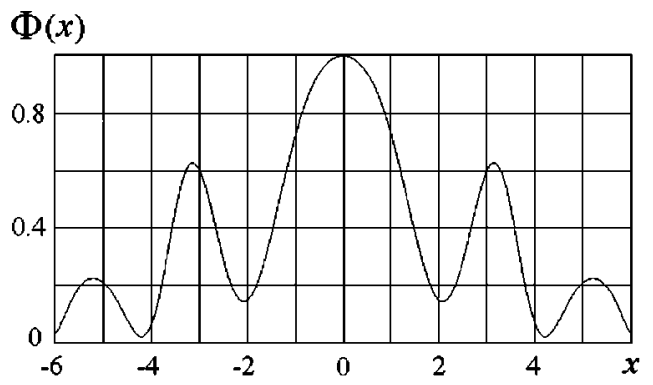

(a)

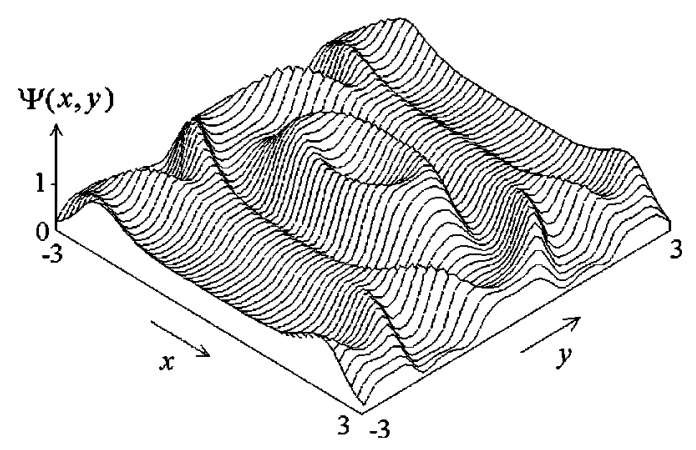

(b)

FIG. 5. Plots of the eigenfunctions for Eq. (16), eigenvalue $\Omega$ $=\gamma^{2}=43.81164 \ldots$ (a), and for Eq. (17), eigenvalue $\Theta=\nu^{2}$ $=7.36414 \ldots$ (b). 
Here $\left\{h_{1}, h_{12}\right\}$ and $\left\{0, h_{2}\right\}$ represent eigenvectors of the linearized $R G$ equation without noise associated with the eigenvalues $\delta_{1}=4.6692 \ldots$ and $\delta_{2}=2.3927 \ldots$, respectively. The coefficients $C_{1}$ and $C_{2}$ depend on the parameters and vanish at the bicritical point. In a close neighborhood of the critical point it is sufficient to account only for the leading terms of the expansions and set $C_{1} \propto\left(\lambda-\lambda_{c}\right), C_{2} \propto(A$ $-A_{c}$ ).

Now let us formulate the basic scaling property that follows from Eqs. (22). If we double the number of time steps (i.e., change $k$ to $k+1$ ), decrease parameter differences $\Delta \lambda$ $=\lambda-\lambda_{c}$ and $\Delta A=A-A_{c}$ by factors $\delta_{1}$ and $\delta_{2}$, respectively, and decrease noise amplitudes $\kappa$ and $\varepsilon$ by factors $\gamma$ and $\nu$, then the form of the stochastic map [Eq. (22)] remains unchanged. Thus, with the new parameters, the noisy system will demonstrate the same behavior as with the old ones, but with twice the time scale.

\section{SCALING PROPERTIES FOLLOWING FROM THE RENORMALIZATION GROUP ANALYSIS AND THEIR DEMONSTRATION IN NUMERICAL COMPUTATIONS}

Now we intend to discuss some manifestations of the effects of noise on dynamics of the unidirectionally coupled maps at the bicritical point and in its vicinity in view of the scaling properties stated in Sec. IV.

\section{A. Noisy bicritical attractor}

Without noise the attractor at the bicritical point is a multifractal object embedded in two-dimensional phase space (with an estimated fractal dimension of 1.0785) [12]. In the presence of noise, the subtle structure of the attractor is smeared out level by level as the intensity of noise grows. In accordance with the conclusions of Sec. IV, each new level of the structure blurs when we increase the magnitude of the noise source in the master system by a factor $\gamma$ and the magnitude of the noise source in the slave system by a factor $\nu$. Figures 6 and 7 show portraits of the attractor of our model system (5) at the bicritical points $\lambda=\lambda_{c}$ $=1.401155 \ldots, A=A_{\mathrm{c}}=1.12498 \ldots$, and $B=0.375$ on the plane of dynamical variables $(x, y)$ in the presence of noise.

Figure 6 relates to the case of noise added only to the master system. The top row corresponds to the noise intensity $\kappa_{0}=0.0001$, and the bottom row to $\kappa_{0} \gamma$. Each subsequent plot in a row shows a box from the previous diagram under magnification by factors $\alpha$ and $\beta$ along the horizontal and vertical axes, respectively. (With an inversion, because of the negativity of both factors.) Figure 7 presents analogous diagrams for the case when the noise source is added only to the slave system. Here the intensity of noise is $\varepsilon_{0}$ $=0.04$ for the top row, and $\varepsilon_{0} \nu$ for the bottom row. On both figures one can observe that diagrams (c), (f), (d), and (g) look remarkably similar in agreement with the expected scaling property.

Visual comparison of Figs. 6 and 7 demonstrates an evident qualitative difference with respect to the effects of noise added to the master and to the slave subsystem. In the first case the structure is blurred both in the longitudinal and transversal directions; in the second case the transversal fractal structure continue to exist in spite of the action of noise.

It is worth stressing that quantitatively the effects of noise on the bicritical attractor are much stronger in the case when the noise source is added to the master system than when it is added to the slave system. (Indeed, a comparable degree of blur in the attractor structure on Figs. 6 and 7 corresponds to $\varepsilon_{0} / \kappa_{0}=400$.) This circumstance is linked, obviously, with the fact that the scaling constant for noise intensity is essentially larger for the first situation.

\section{B. Effects of noise on the Fourier spectrum}

At the bicritical point both the master and slave systems generate Fourier spectra that are hierarchically organized and contain components at frequencies $m / 2^{k}$ with regularly decreasing intensities in dependence on the level number $k$. In the master subsystem this is the well-known spectrum associated with the Feigenbaum criticality [22]. In the slave subsystem the spectrum is qualitatively similar, but quantitatively distinct $[11,12]$ : the decrease of the spectral intensities from level to level is much slower. Figure 8 illustrates a change of the spectrum generated by the slave system in the presence of noise in the master system. Each subsequent plot corresponds to a larger intensity of noise. Figure 9 presents analogous spectra for the case when the noise is added only to the slave system. The observed effect of subsequent smearing of subtle details of the spectral structure from level to level is more or less obvious, but we find it useful to present here the spectra because they could be directly compared with those obtained in physical experiments. Again we point out that influence of the noise added to the master system is stronger than that of noise in the slave system.

\section{Lyapunov exponent in the presence of noise}

In accordance with the results of Sec. IV, at the bicritical point the system will demonstrate similar behaviors for the noise intensity parameters $(\kappa, \varepsilon)$ as for $(\kappa / \gamma, \varepsilon / \nu)$, but with a doubled characteristic time scale in the second case. Thus the magnitudes of the Lyapunov exponents at $(\kappa / \gamma, \varepsilon / \nu)$ must be half the magnitude at $(\kappa, \varepsilon)$. Hence we may estimate critical indices for the Lyapunov exponents with respect to the intensities of noise sources added to both subsystems. That is, they must behave as

$$
\Lambda_{1} \cong c_{0} \kappa^{\rho} \quad \text { and } \quad \Lambda_{2} \cong c_{1} \kappa^{\rho}+c_{2} \varepsilon^{v},
$$

where $\quad \rho=\log _{\gamma} 2=0.36675 \ldots, v=\log _{\nu} 2=0.69432 \ldots$, and $c_{0}, c_{1}$, and $c_{2}$ are some coefficients.

The scaling properties of the Lyapunov exponents are demonstrated in Fig. 10. We show the plots for the both exponents associated with the master and slave subsystems, $\Lambda_{1}$ and $\Lambda_{2}$, versus the parameter of noise intensity $\kappa$ with $\varepsilon=0$ [(a) and (b)], and the plot for $\Lambda_{2}$ exponent versus the noise parameter $\varepsilon$ with $\kappa=0$ (c). A selected box is shown with magnification by a factor 2 along the vertical axis, and by a factor $\gamma[(\mathrm{a})$ and (b)] or $\nu$ (c) along the horizontal axis. 

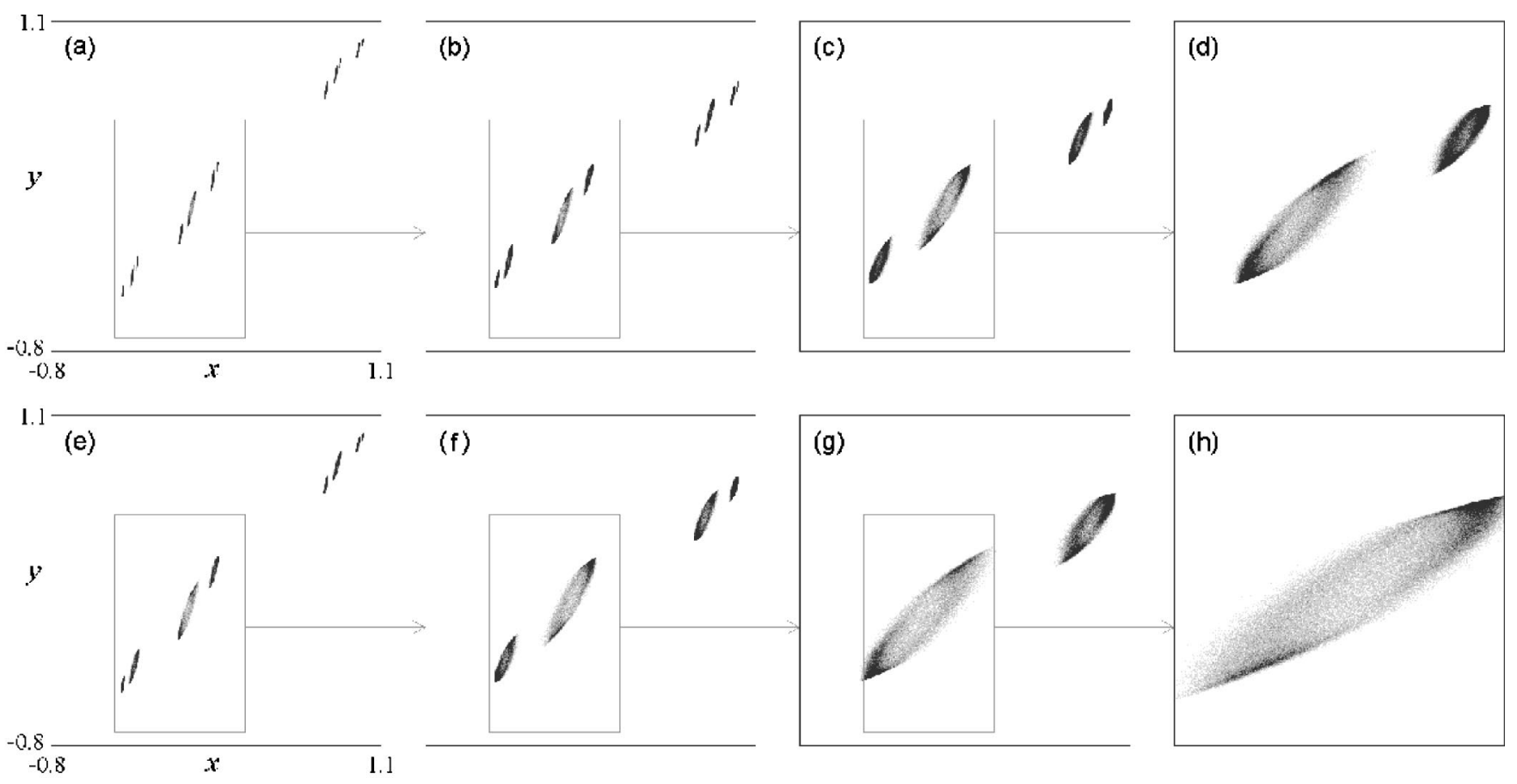

FIG. 6. Portraits of attractors on the phase plane $(x, y)$ in the presence of noise in the master subsystem only; $\lambda=\lambda_{c}, A=A_{c}, B$ $=0.375$, and $\varepsilon=0, \kappa=0.0001$ for (a) $-(\mathrm{d})$, and $\kappa=0.0001 \gamma$ for (e)-(f). Each subsequent diagram of the top and bottom rows reproduces a selected box from the previous plot under magnification by factors $\alpha$ and $\beta$ along the horizontal and vertical axes, respectively. Compare (c) with (f) and (d) with (g) to observe scaling.
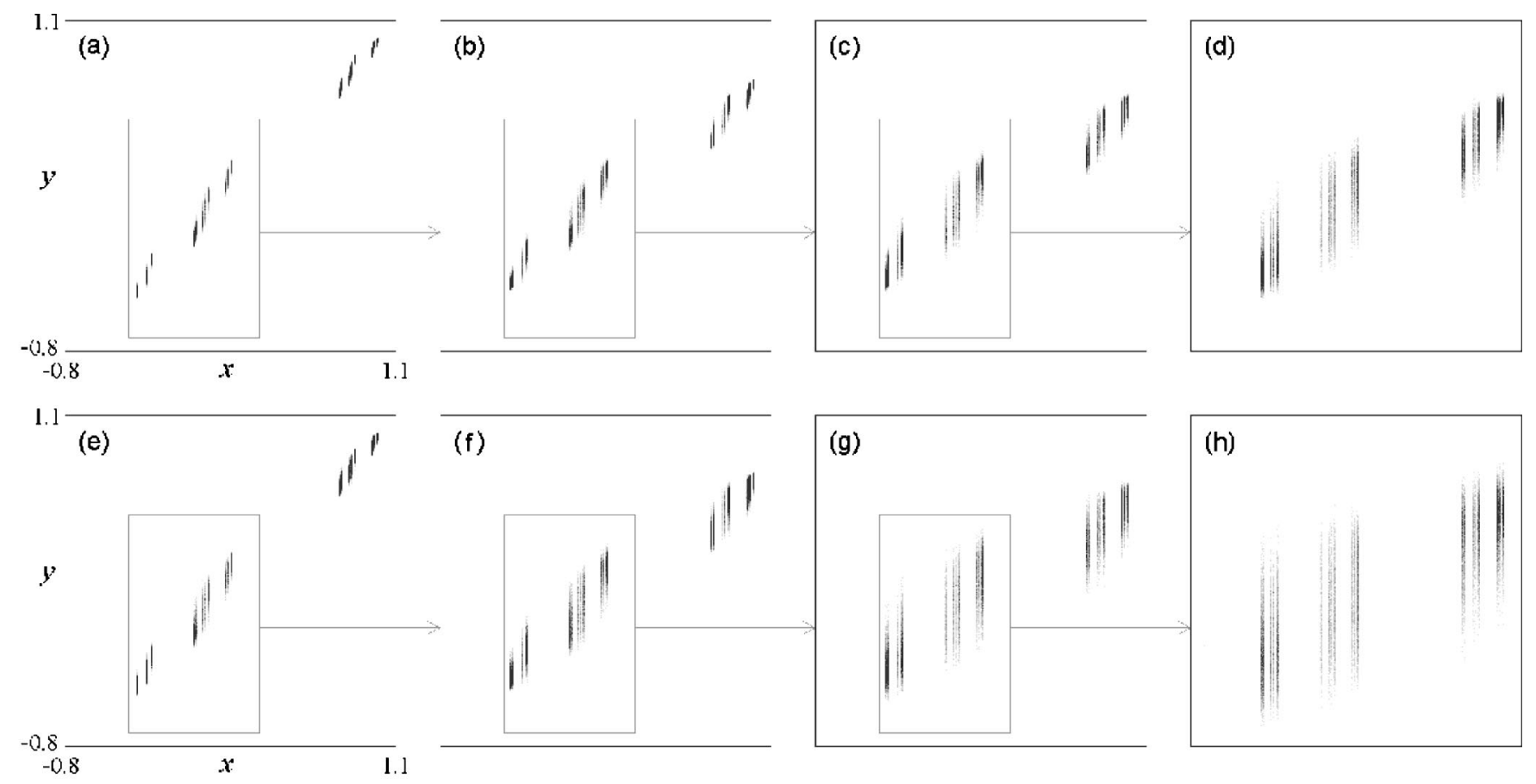

FIG. 7. Portraits of attractors on the phase plane $(x, y)$ in the presence of noise in the slave subsystem only; $\lambda=\lambda_{c}, A=A_{\mathrm{c}}, B$ $=0.375, \kappa=0$, and $\varepsilon=0.04$ for (a)-(d), and $\varepsilon=0.04 \nu$ for (e)-(f). Each subsequent diagram of the top and bottom rows reproduces a selected box from the previous plot under magnification by factors $\alpha$ and $\beta$ along the horizontal and vertical axes, respectively. Compare (c) with (f) and (d) with (g) to observe scaling. 


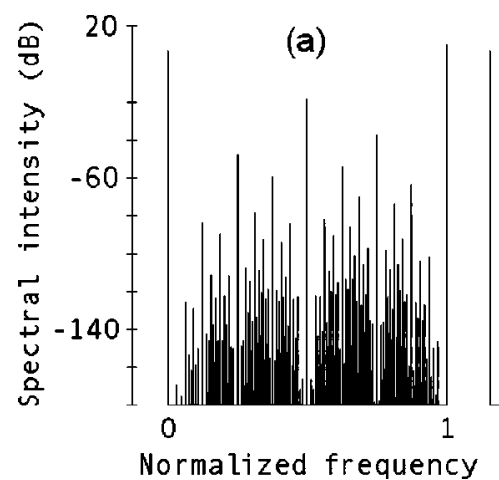

(b)

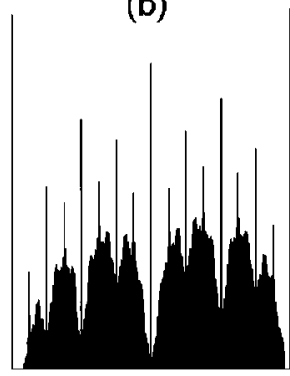

(c)

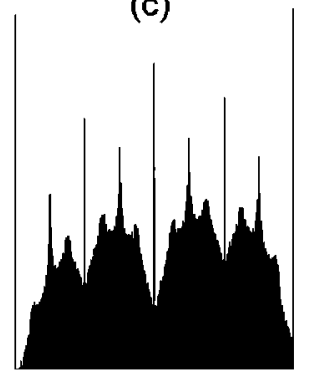

(d)

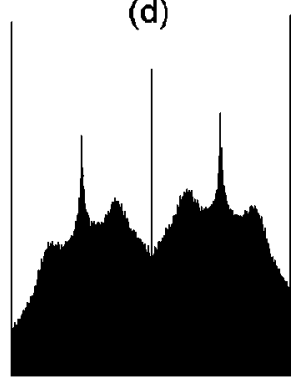

FIG. 8. Fourier spectra generated by the slave system at the bicritical point in the absence of noise (a) and in the presence of a noise source in the master system at $\kappa=\kappa_{0}=0.00006(\mathrm{~b}), \kappa=\kappa_{0} \gamma(\mathrm{c})$, and $\kappa=\kappa_{0} \gamma^{2}$ (d).

Observe the self-similarity of the pictures under this scale change.

It is worth emphasizing that the effects of noise on the master and on the slave subsystem are of opposite character: In the master system the Lyapunov exponent becomes greater in the presence of noise, while in the slave system it decreases. [In other words, the coefficient $c_{0}$ in Eq. (22) is positive, while $c_{1}$ and $c_{2}$ are negative.]

\section{Self-similar arrangement of a vicinity of the bicritical point in the parameter plane for the noisy system}

In the presence of noise the structures visible on the Lyapunov chart become more and more blurred as the noise intensity grows [cf. Figs. 2 and 1(b)]. On the basis of the scaling arguments of Sec. IV, we can now reveal a quantitative aspect of this process. In Fig. 11 we show the Lyapunov charts for close neighborhoods of the bicritical point, which is located exactly at the center of each plot. Diagram (a) relates to the case of the noise source in the master subsystem. The first panel (a) corresponds to the noise intensity parameter $\kappa=\kappa_{0}=0.01$, and the next two panels to $\kappa$ $=\kappa_{0} / \gamma$ and $\kappa=\kappa_{0} / \gamma^{2}$, respectively, with magnification along the horizontal and vertical axes using the factors $\delta_{1}$ and $\delta_{2}$. For the three pictures in the row the gray tones code values of $\Lambda_{2}, 2 \Lambda_{2}$, and $4 \Lambda_{2}$ to illustrate rescaling of the Lyapunov exponent, and to make the similarity of the structures clearly visible. Panel (b) supplies analogous diagrams for the case when the noise source is in the slave subsystem. The first picture corresponds to $\varepsilon=\varepsilon_{0}=0.06$, and the two others to $\varepsilon_{0} / \nu$ and $\varepsilon_{0} / \nu^{2}$. Observe the excellent correspondence of the charts in each row.

\section{E. Scaling indices for a parameter shift of the corner point of the region of hyperchaos}

As we mentioned, in the presence of noise the Lyapunov exponent in the master system grows, while in the slave system it falls. To restore the situation of the chaos threshold, i.e., that of zero Lyapunov exponents, we should decrease the control parameter of the master system and increase that in the slave system. In Fig. 12 we present a chart of the parameter plane for a system of two unidirectionally coupled maps [Eqs. (5)] with the noise intensity parameters $\kappa=\varepsilon=0.05$. The areas marked "Ch-I" and "Ch-II" correspond to the presence of a positive Lyapunov exponent in one of the subsystems, either in the master or in the slave system. The area of two positive exponents (hyperchaos) is designated as "Hyper." Around the figure we present a set of so-called snapshot phase portraits (see Ref. [23]) at representative points of the parameter plane. Such a portrait is a set of points on the plane $(x, y)$ depicting instantaneous states of a large number of identical systems (an ensemble), subjected to identical realizations of the noise. Using the scaling properties of the system in the presence of noise, it is possible to obtain an estimate of the shift of the corner point of the

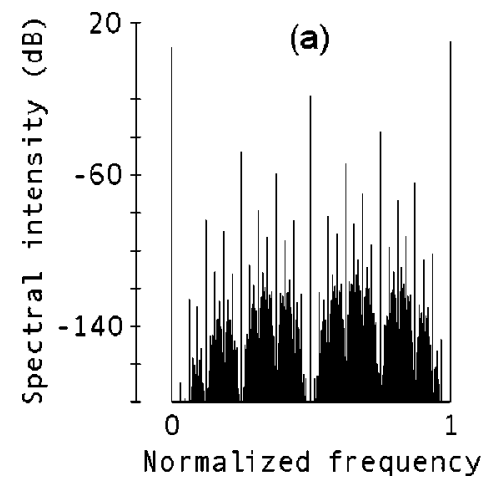

(b)

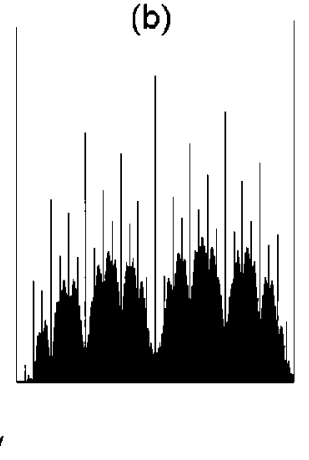

(c)

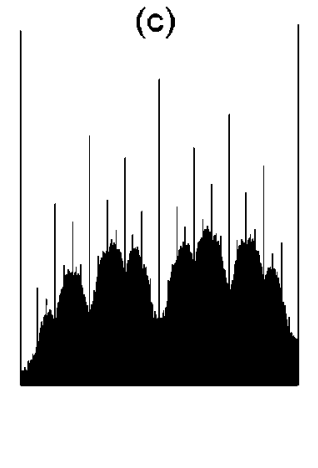

(d)

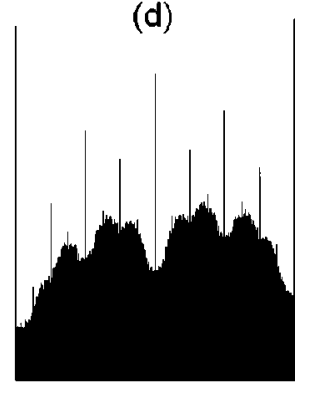

FIG. 9. Fourier spectra generated by the slave system at the bicritical point in presence of a noise source in the slave system at $\kappa=0$ and $\varepsilon=\varepsilon_{0}=0.001$ (a), $\varepsilon=\varepsilon_{0} \nu$ (b), $\varepsilon=\varepsilon_{0} \nu^{2}(\mathrm{c})$, and $\varepsilon=\varepsilon \nu^{3}$ (d). 

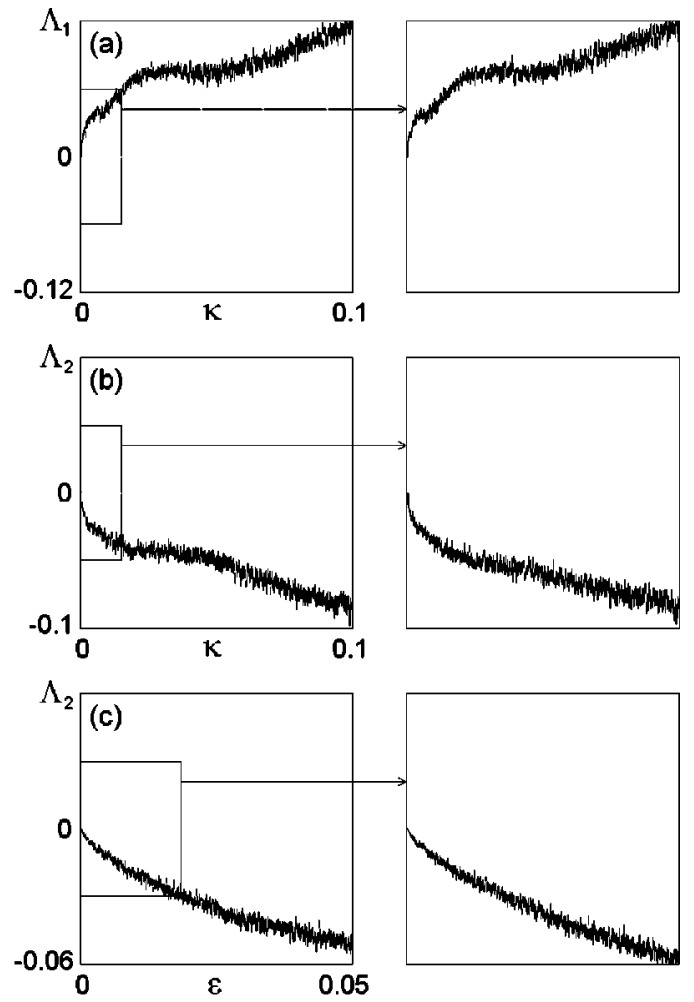

FIG. 10. Lyapunov exponents vs noise intensity at the bicritical point $\lambda=\lambda_{c}, A=A_{c}$, and $B=0.375$ : (a) and (b) with the noise source in the master map, and (c) in the slave map. Observe that the self-similarity of the pictures under scale changes with factor 2 along the vertical axes, and with factors $\gamma=6.619$ [diagrams (a) and (b)] and $\nu=2.713$ [diagram (c)] along the horizontal axes.

region of hyperchaos from the bicritical point. That is, if a source of noise is present only in the master system, the displacement of the hyperchaos corner from the bicritical point will behave as
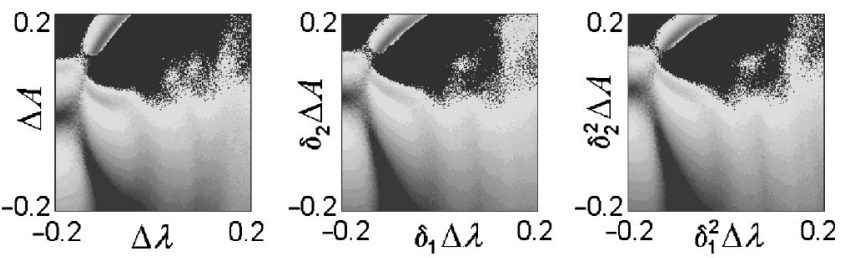

(a)
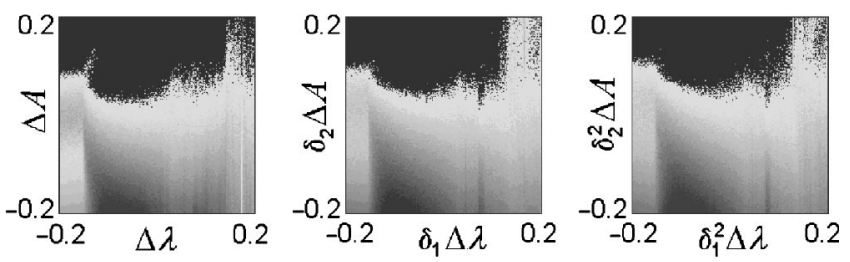

(b)

FIG. 11. Lyapunov charts demonstrating scaling in a neighborhood of the bicritical point: (a) the noise source in the master subsystem, and (b) in the slave subsystem. The first plot (a) corresponds to the noise intensity parameter $\kappa=0.01$, which is decreased by factor $\gamma=6.619 \ldots$ for each next diagram in the row. The first diagram (b) corresponds to $\varepsilon=0.06$, and others to the values subsequently rescaled by $\nu=2.713 \ldots$ The gray scale coding for the Lyapunov exponent $\Lambda_{2}$ is redefined at each new level of magnification to make the similarity clearly visible.

$$
\Delta \lambda \propto \kappa^{a}, \quad \Delta A \propto \kappa^{b},
$$

where

$$
a=\log _{\gamma} \delta_{1} \cong 0.815359 \text { and } \quad b=\log _{\gamma} \delta_{2} \cong 0.461617
$$

If the source of noise is added only to the slave system, then

$$
\Delta \lambda=0, \quad \Delta A \propto \kappa^{c},
$$
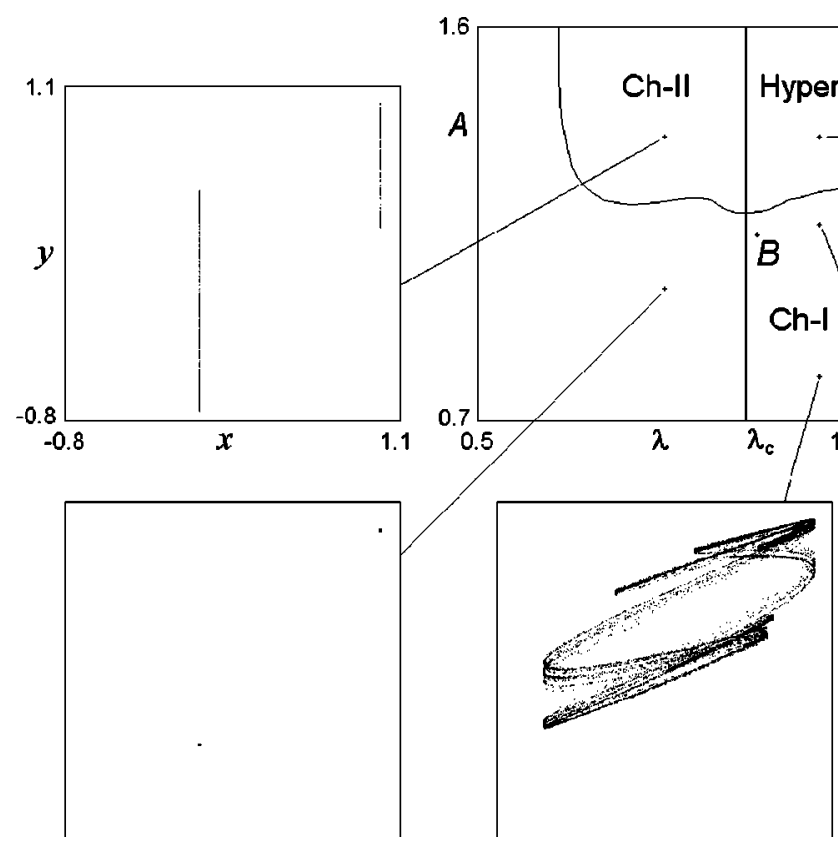

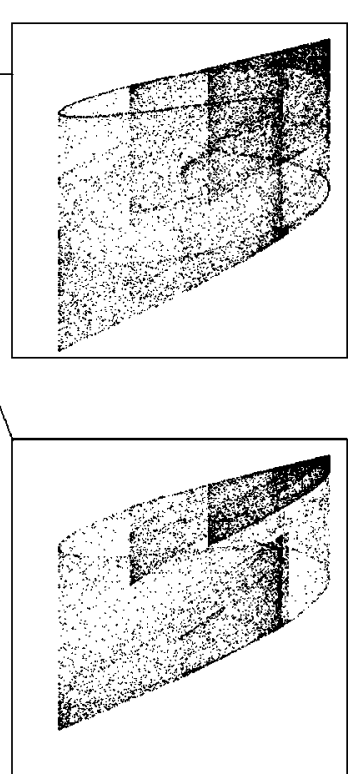

FIG. 12. Snapshot phase portraits at some representative points of the parameter plane for an ensemble of about $10^{5}$ copies of the system effected by an identical sample of the noise after 768 iterations. Initial conditions were chosen randomly from the square $-1 \leqslant x \leqslant 1,-1 \leqslant y \leqslant 1$. Noise is present both in the master and slave subsystems, $\kappa=\varepsilon=0.05$. The marks Ch-I and Ch-II designate regimes with one positive Lyapunov exponent relating to the master and slave subsystems, and Hyper designates the regime of two positive Lyapunov exponents and hyperchaos in the noisy system. The bicritical point found in absence of noise is marked as $B$. 

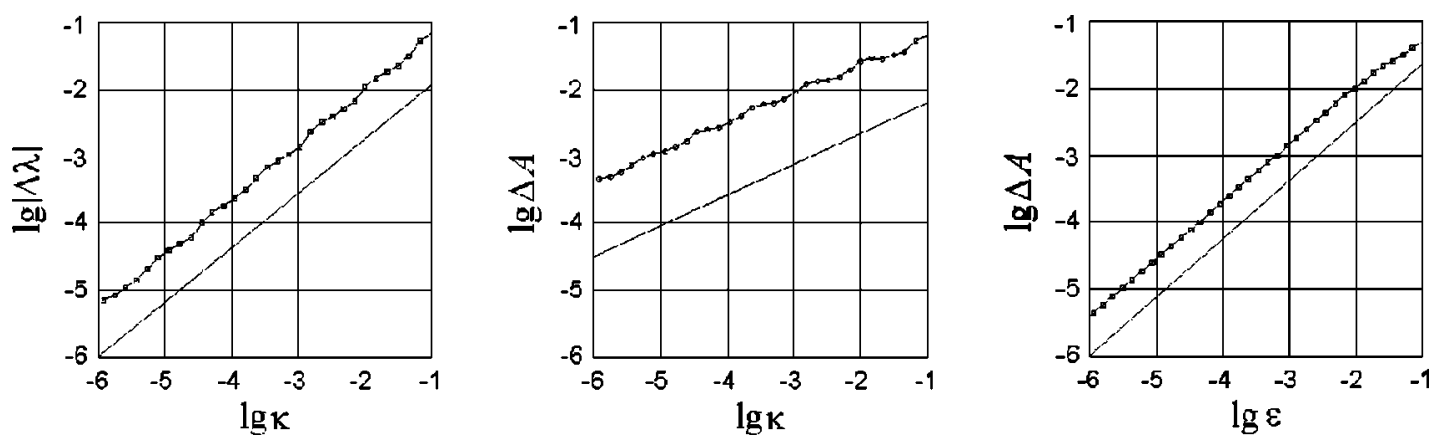

FIG. 13. Shift of the parameters $\lambda$ and $A$ vs the intensity of noise added in the master [(a) and (b)] and in the slave subsystem (c) on a double logarithmic scale. The straight lines show the slopes corresponding to the power-law estimates [Eqs. (24) -(27)].

where

$$
c=\log _{\nu} \delta_{2} \cong 0.873909 \text {. }
$$

The double-logarithm plots shown in Fig. 13 present data of computations supporting these scaling estimates. The straight lines are drawn with a slope corresponding to the predicted critical indices [Eqs. (24) and (27)].

\section{CONCLUSION}

We have discussed scaling regularities associated with the effect of additive noise on the bicritical behavior in the system of two unidirectionally coupled quadratic maps, both of which are brought to the threshold of chaos by adjustment of their two control parameters. A renormalization group analysis of the effect of noise was developed, and respective universal functions and constants were computed. We outlined the qualitative and quantitative differences between the response with respect to random perturbations added to the master system and to the slave system. In particular, the universal constants determining the rescaling rules for the parameters of the intensity of the noise sources in the master and slave subsystems were found to be $\gamma=6.619036 \ldots$ and $\nu=2.713708 \ldots$, respectively. We also presented a number of computer graphical illustrations for the scaling regulari- ties. In particular, we paid attention to the smearing of the fine structure of the bicritical attractor and the Fourier spectra due to the presence of noise. We discussed the self-similar structure of the Lyapunov charts on the parameter plane near the bicritical point, and the shift of the hyperchaos threshold in dependence of the noise intensity.

We considered a particular representative of the universality class (two coupled one-dimensional maps). Nevertheless, on the basis of RG argumentation, we may conjecture that the same regularities will be intrinsic to a wide class of systems composed of two period-doubling subsystems with unidirectional coupling. We expect that these results will be helpful for experimental researches aimed at the observation and investigation of bicritical behavior in systems of different physical natures. In this connection, we emphasize that the influence of noise is much stronger when the noise is added to the master system than to the slave system. Hence in the experiments one should pay particular attention to the reduction of noise in the master system.

\section{ACKNOWLEDGMENTS}

The work was performed under a partial support from Russian Foundation of Basic Researches (Grant Nos. 00-0217509 and 01-02-06388). J.K. and A.K. acknowledge support from CRDF, award REC-006. S.K. acknowledges the DTU for financial support.
[1] M. J. Feigenbaum, J. Stat. Phys. 19, 25 (1987); 21, 669 (1979); Physica D 7, 16 (1983).

[2] S. J. Chang, M. Wortis, and J. A. Wright, Phys. Rev. A 24, 2669 (1981); B. Hu and J. Rudnik, Phys. Rev. Lett. 48, 1645 (1982); S. J. Shenker, Physica D 5, 405 (1982); M. J. Feigenbaum, L. P. Kadanoff, and S. J. Shenker, Physica D 5, 370 (1982); D. Rand, S. Ostlund, J. Sethna, and E. D. Siggia, Phys. Rev. Lett. 49, 132 (1982).

[3] A. P. Kuznetsov, S. P. Kuznetsov, and I. R. Sataev, Physica D 109, 91 (1997).

[4] J. P. Crutchfield, M. Nauenberg, and J. Rudnik, Phys. Rev. Lett. 46, 933 (1981); B. Shraiman, C. E. Wayne, and P. C. Martin, ibid. 46, 935 (1981).

[5] J. E. Hirsch, B. A. Huberman, and D. J. Scalapino, Phys. Rev.
A 25, 519 (1981); J. E. Hirsch, M. Nauenberg, and D. J. Scalapino, Phys. Lett. 87A, 391 (1982); J. P. Eckmann, L. Thomas, and P. Wittwer, J. Phys. A 14, 3153 (1981).

[6] A. Hamm and R. Graham, Phys. Rev. A 46, 6323 (1992).

[7] G. Györgyi and N. Tishby, Phys. Rev. Lett. 58, 527 (1987).

[8] G. Györgyi and N. Tishby, Phys. Rev. Lett. 62, 353 (1989).

[9] K. Kaneko, Phys. Lett. 111A, 321 (1985); V. S. Anishchenko, I. S. Aranson, D. E. Postnov, and M. I. Rabinovich, Dokl. Akad. Nauk (SSSR) 286, 1120 (1986) [Sov. Phys. Dokl. 31, 169 (1986)]; I. S. Aranson, A. V. Gaponov-Grekhov, and M. I. Rabinovich, Physica D 33, 1 (1988).

[10] L. Kocarev and U. Parlitz, Phys. Rev. Lett. 76, 1816 (1996); M. Hasler, Int. J. Bifurcation Chaos Appl. Sci. Eng. 8, 647 (1998). 
[11] B. P. Bezruchko, Yu. V. Gulyaev, S. P. Kuznetsov, and E. P. Seleznev [Dokl. Akad. Nauk (SSSR) 287, 619 (1986) [Sov. Phys. Dokl. 31, 258 (1986)].

[12] S. P. Kuznetsov, Izv. Vuz. Radiofiz. 33, 788 (1990); A. P. Kuznetsov, S. P. Kuznetsov, and I. R. Sataev, Int. J. Bifurcation Chaos Appl. Sci. Eng. 1, 839 (1991); S. P. Kuznetsov and I. R. Sataev, Phys. Lett. A 162, 236 (1992).

[13] S. Y. Kim, Phys. Rev. E 59, 6585 (1999).

[14] A. P. Kuznetsov, S. P. Kuznetsov, I. R. Sataev, and L. O. Chua, Int. J. Bifurcation Chaos Appl. Sci. Eng. 6, 119 (1996).

[15] S.-Y. Kim and W. Lim, Phys. Rev. E 63, 036223 (2001).

[16] A. P. Kuznetsov, S. P. Kuznetsov, and I. R. Sataev, Tech. Dig. Ser.-Opt. Soc. Am. 16, 209 (1992).

[17] B. P. Bezruchko and O. B. Pudovochkin, Izv. Vuz. Radiofiz. 35, 39 (1992).
[18] A. P. Kuznetsov, S. P. Kuznetsov, and I. R. Sataev, Int. J. Bifurcation Chaos Appl. Sci. Eng. 3, 139 (1993).

[19] J. Rössler, M. Kiwi, B. Hess, and M. Markus, Phys. Rev. A 39, 5954 (1989); M. Marcus and B. Hess, Comput. Graph. 13, 553 (1989); J. C. Bastos de Figueireido and C. P. Malta, Int. J. Bifurcation Chaos Appl. Sci. Eng. 8, 281 (1998).

[20] D. Fiel, J. Phys. A 20, 3209 (1987).

[21] O. E. LanfordIII, Bull. Am. Math. Soc. 6, 427 (1982).

[22] M. J. Feigenbaum, Phys. Lett. A 74, 375 (1979); M. Nauenberg and J. Rudnik, Phys. Rev B 24, 493 (1981); B. Huberman and A. Zisook, Phys. Rev. Lett. 26, 626 (1981); A. Pikovsky, Radiophys. Quantum Electron. 29, 1076 (1985).

[23] F. J. Romeiras, C. Grebogi, and E. Ott, Phys. Rev. A 41, 784 (1990). 